\title{
HEMIORCHIS RHODORRHACHIS SCHUM. (ZINGIBERACEAE) - A NEW RECORD FOR BANGLADESH
}

\author{
S. C. SRivastava and P. P. Ghoshal \\ Botanical Survey of India, P.O. Botanic Garden, Howrah-711 103, \\ West Bengal, India
}

Key words: Hemiorchis rhodorrhachis, new record, Bangladesh

During a taxonomic study of the genus Hemiorchis Kurz (Zingiberaceae) in India and its vicinity, a specimen of Lister deposited at CAL was found to be wrongly identified as $H$. pantlingii King. The correct identity of the specimen significantly influenced its known distribution. So far the species is known to occur from India (Meghalaya) and Burma, but the present specimen of Lister, from Barkal. establishes its occurrence in Bangladesh too. The present paper provides detailed description along with an analytical illustration for easy identification.

The species is closely allied to $H$. pantlingii King, but differs in having the corolla tube almost equal to clayx. A detailed description and illustration of the species for easy identification is given here.

Hemiorchis rhodorrhachis Schum. in Engler, Das pflanzenreich, heft 20: 128 (1904). Rao et Verma in Bull. Bot. Surv. India 14 (1-4): 119 (1972); Mitra, Flow. Pl. East. India 1: 249, 1958. H. burmanica auct. non Kurz 1873; Baker in Curtis, Bot. Mag. 46 ser. 3. t. 7120. 1890 et in Hook. f., Fl. Brit. India 6: 2071890. Type: India: Khasia hill, G. Mann. 1889 (K, n.v.).

(Fig. 1)

Herb, terrestrial, 10-15 cm tall. Rhizome vertical, cylindrical, muddy white outside, creamy white inside, c. $0.5 \mathrm{~cm}$ thick. Leaves 3-6, produced after flowering, sheathing the stem; lamina oblong-lanceolate, entire, membranous margin, dark green above, pale green beneath, acute apex, pubescent. Inflorescence radical, spike c. $12 \mathrm{~cm}$ long; peduncle covered with spathe; spathe 5-7, 4.0-6.2 cm long, round at tip, pink, entire margin, puberulous; flower spirally arranged, ebracteate, orange coloured, $1.8-2.2 \mathrm{~cm}$ long, 1-2 flowers open at a time. Calyx 7-8 $\times 4-5 \mathrm{~mm}$, tubular, trifid apically, pink, pubescent; sinus c. $1.5 \mathrm{~mm}$; lobes slightly bifid. Corolla tube c. $8 \mathrm{~mm}$ long, orange. Lateral petals c. $12 \times 5 \mathrm{~mm}$, membranous, cream coloured, oblong-ovate, entire margin, hispidulous, obtuse apex, 3-nerved; dorsal lobe $12 \times 7 \mathrm{~mm}$, cream coloured, membranous, broadly oblong-ovate, entire margin, hispidulous, obtuse apex, 3-nerved. Staminodes c. 12 - $7 \mathrm{~mm}$, bit leathery, obliquely ovate-oblong, orange or yellow, entire margin, obtuse apex, 3-nerved, slightly clawed; claw c. $3 \mathrm{~mm}$. Lip $14 \times 10 \mathrm{~mm}$, broadly ovate or orbicular when open, yellowish or orange, spotted with red-brown dots, leathery, 


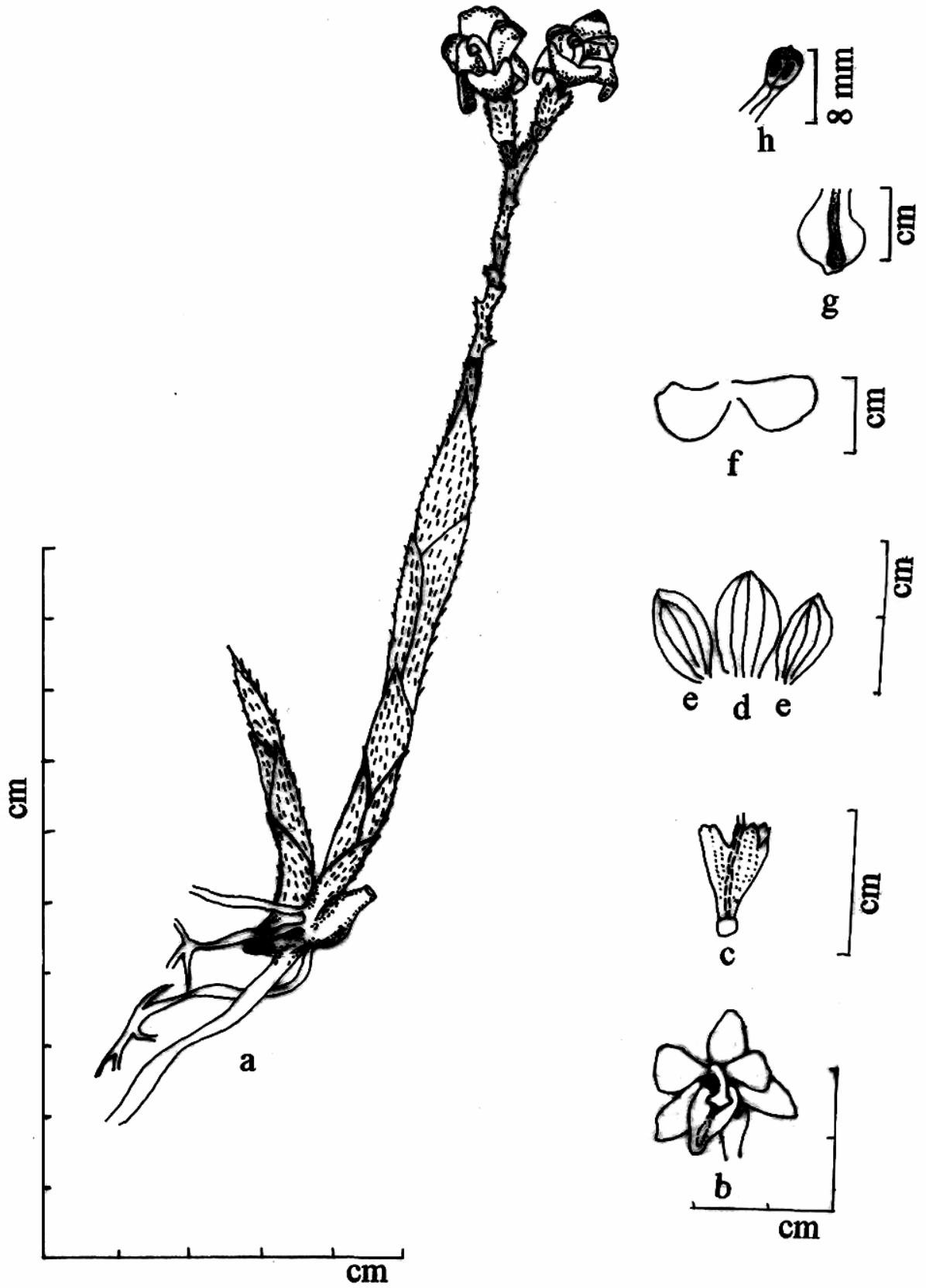

Fig. 1. a-h: Hemiorchis rhodorrhachis Schum. a. habit; b. flower, c. calyx; d. dorsal petal; e. lateral petals; f. staminodes; g. lip and h. stamen.

entire margin, deep orange midrib like appearance which is protruding out in beak shape structure. Stamen c. $8 \mathrm{~mm}$ long, anther c. 3-4 mm long, apiculate, yellow. Stigma rounded, pubescent, protuded out of anther. Ovary c. $2 \mathrm{~mm}$ long, oblong, tomentose. 
HEMIORCHIS RHODORRHACHIS (ZINGIBERACEAE)

Specimens examined: Bangladesh: Chittagong Hill Tracts, Barkal, 1876, J.L. Lister 305 (CAL).

\section{References}

Baker, J.G. 1890. In: Curtis's Bot. Mag. 46, ser. 3, t. 7120. L. Reeve \& Co., 5 Henrietta St., Covent Garden, London.

Baker, J.G. 1890. In: Hooker, J.D. (ed.) Fl. Brit. India 6: 206-207. L. Reeve \& Co., Ltd. The Oast House, Brook, Ashford, Kent.

Mitra, J.N. 1958 Flowering Plants of Easten India 1 - Monocotyledons. 249-250. The world Pres Private Ltd. Calcutta.

Rao, A.S. and D.M. Verma. 1972. Materials for a monocot Flora of Assam - II (Zingiberaceae and Marantaceae) in Bull. Bot. Sur. India 14(4): 119.

Scumann, K. 1904. In: Engler, A. (ed.) Das Pflanzenreich Regni Vegetabilis Conspectus.। heft 20: 127-129 Im Verlag von H.R. Engelmann (J. Cramer) Weinheim/BergstrafBe. 